\title{
Obesity and its association with Chronic Periodontitis - A pilot study
}

\author{
Dr. Shashikanth Hegde ${ }^{1}$, Dr. Elashri ${ }^{2}$, Dr. Rajesh Kashyap. $S^{3}$, \\ Dr. Arun Kumar M.S ${ }^{4}$ \\ ${ }^{1}$ (Prof \& Head, Dept. of Periodontics, Yenepoya dental College \& Hospital, Mangalore, India) \\ ${ }_{2}^{2}$ (Post Graduate student, Dept. of Periodontics, Yenepoya dental College \& Hospital, Mangalore, India) \\ ${ }^{3}$ (Professor, Dept. of Periodontics, Yenepoya dental College \& Hospital, Mangalore, India) \\ ${ }^{4}$ (Professor, Dept. of Periodontics, Yenepoya dental College \& Hospital, Mangalore, India)
}

\begin{abstract}
:
Aim: To study the association of obesity with the severity of periodontitis and to compare the blood glucose levels and plasma lipid profile in obese and non obese subjects with chronic periodontitis. Materials and Methods: A total of 20 systemically healthy patients, aged 30 to 60 years were included in the study. Periodontal status of the subjects was assessed by recording Community Periodontal Index (CPI) and Gingival Index (Loe\& Silness 1963). Body mass index (BMI) and waist circumference (WC) were used as measures to assess obesity. The fasting plasma lipids level and fasting blood glucose level were measured. Statistical Analysis: Data were analyzed by unpaired 't' test, Fishers Exact Test, Chi-square test using SPSS version 17 software. A p-value of 0.05or less was considered to be significant. Results: Gingival index was significant in obese group with chronic periodontitis compared to non obese group with chronic periodontitis $(p=0.001)$. Higher probing depth and loss of attachment in obese group compared to the non obese group whereas it did not reach statistical significance ( $p=0.517$ ). The mean fasting lipid profile showed significantly higher total cholesterol, triglycerides, $L D L$ and VLDL levels $(p<0.05)$ in obese group.
\end{abstract}

Keywords: obesity, periodontitis, body mass index (BMI), gingival index, lipid profile.

\section{Introduction}

Obesity represents a condition wherein the proper sensing and management of nutrients and energy status present significant challenges at cell and organism levels. The organism cannot adapt and maintain homeostasis under continuous energy and nutrient exposure, and the consequent emergence of metabolic and oxidative stress leads to inflammatory responses and cell organelle dysfunction. ${ }^{[1]}$ Obesity is often associated with increased lipid and blood glucose levels, which may have deleterious consequences for the host response leading to alterations in $\mathrm{T}$ cells and monocytes/macrophages, as well as an increased cytokine production. This imbalance in the host immune system may increase the risk for infection. ${ }^{[2]}$

Periodontitis is an inflammatory disease process that results in permanent destruction to tissues of the periodontium, including the loss of gingival connective tissue, destruction of the periodontal ligament and resorption of alveolar bone. ${ }^{[3]}$ It is associated with increased prevalence of cardiovascular morbidity and mortality. There is a rationale that indicates that the presence of periodontal pockets which can harbor pathogenic microorganisms can evoke a host response and elicit a systemic effect. ${ }^{[4]}$

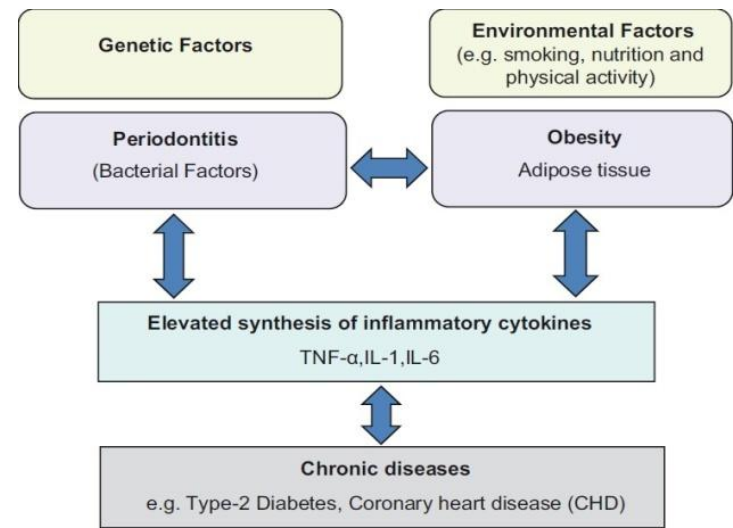

Fig 1. Model linking periodontitis and obesity with inflammatory related chronic diseases 
Relationship among periodontitis, obesity, and chronic diseases may be multidirectional [Fig.1] ${ }^{[5-6]}$ Inflammatory diseases like periodontitis induce the production of pro-inflammatory cytokines such as TNF- $\alpha$, IL-1, and IL-6. ${ }^{[7-8]}$ It has been suggested that the secretion of TNF- $\alpha$ by adipose tissue triggered by LPS (lipopolysaccharides) from periodontal Gram- negative bacteria promotes hepatic dyslipidemia and decreases insulin sensitivity. ${ }^{[9-10]}$ These suggest a potential interaction among obesity, periodontitis, and chronic disease incidence. This study was taken up to ascertain the association of obesity with the severity of periodontitis and to compare the blood glucose levels and plasma lipid profile in obese and non obese subjects with chronic periodontitis.

\section{Methodology}

The present study was conducted in the Department of Periodontology, Yenepoya dental college, Mangalore. The study was approved by Yenepoya University Ethical Committee and informed consent was obtained from all the patients. A total of 20 systemically healthy patients, aged 30 to 60 years reporting to the Department of Periodontology were included in the study. The study population includes both the genders. According to the BMI classification for adult Asians ${ }^{[1]}$ the patients with BMI $\geq 25 \mathrm{~kg} / \mathrm{m}^{2}$ have been grouped into obese category (Group 1) and the with BMI $\leq 24.9 \mathrm{~kg} / \mathrm{m}^{2}$ have been grouped into non obese category (Group 2).

\section{Inclusion criteria:}

Group I (obese) - patients with BMI $\geq 25 \mathrm{~kg} / \mathrm{m}^{2}$ with chronic periodontitis (probing depth $\geq 4 \mathrm{~mm}$ and/or attachment loss $>3 \mathrm{~mm}$ in at least $30 \%$ teeth in oral cavity).

Group II (non obese) - patients with BMI $\leq 24.9 \mathrm{~kg} / \mathrm{m}^{2}$ with chronic periodontitis (probing depth $\geq$ $4 \mathrm{~mm}$ and/or attachment loss $>3 \mathrm{~mm}$ in at least $30 \%$ teeth in oral cavity).

Subjects with any systemic diseases, subjects who were on medication for hypercholesterolemia, smokers, pregnant women or lactating mothers, subjects having history of periodontal surgery in last six months were not included in the study. BMI is computed from weight in kilograms $(\mathrm{kg})$ divided by square height in meters (m). ${ }^{[5]}$ Waist circumference of all the subjects was recorded using measuring tape. According to guidelines of weight classification in adult Asians, cut-offs for waist circumstances will now be $90 \mathrm{~cm}$ for Indian men and $80 \mathrm{~cm}$ for Indian women. ${ }^{[11]}$ Values above these were considered obese. Periodontal status was assessed by using Community Periodontal Index (CPI).The highest CPI score found among all examined sites were concluded as CPI score for that individual. The gingival status was assessed by recording gingival index given by Loe \& Silness (1963). ${ }^{[12]}$ The fasting plasma lipids level and fasting blood glucose level in all the 20 subjects were measured. Fasting Lipid profile was measured using enzymatic method. The enzyme that was used was Cholesterol 0xidase Peroxidase (CHOD-POD). The components of the lipid profile were Total Cholesterol (TC), High Density Lipoprotein (HDL), Low Density Lipoprotein (LDL), very low density lipoprotein (VLDL). Fasting blood glucose level was estimated using Glucose oxidase- Peroxidase (GODPOD) method. Data were analyzed by unpaired ' $t$ ' test, Fishers Exact Test, Chi-square test using SPSS version 17 software. A p-value of 0.05 or less was considered to be significant.

\section{Results}

In this present study, the mean body mass index (BMI) was $35.4 \pm 5.16 \mathrm{~kg} / \mathrm{m}^{2}$ and mean waist circumference (WC) was $98.1 \pm 6.83 \mathrm{~cm}$ in the obese group while the mean BMI in the non obese group was $23.3 \pm 1.39 \mathrm{~kg} / \mathrm{m}^{2}$ and WC was $79.84 \pm 6.65 \mathrm{~cm}$. A statistically significant difference between the two groups was observed using the $t$ test, with higher BMI and higher WC in the obese group $(p<.001)$. The number of males and females did not differ significantly. Gingival index was significant in obese group with chronic periodontitis compared to non obese group with chronic periodontitis $(p=0.001)$. [Fig.2] There is higher probing depth and loss of attachment in obese group compared to the non obese group whereas it did not reach statistical significance $(p=0.517)$. The mean fasting lipid profile showed significantly higher total cholesterol, triglycerides, LDL and VLDL levels ( $\mathrm{p}<0.05$ ). [Fig.3] The differences in HDL and mean fasting blood glucose levels were statistically insignificant in both the groups. 


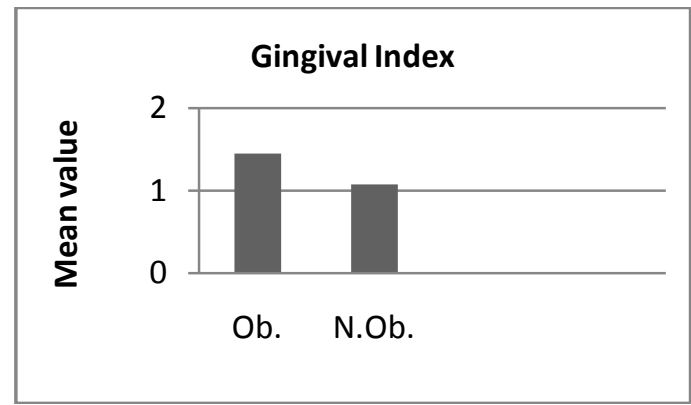

Fig 2. Mean gingival index in obese and non subjects.

$\{\mathrm{Ob} .=$ obese, $\mathrm{N} . \mathrm{Ob} .=$ non obese $\}$

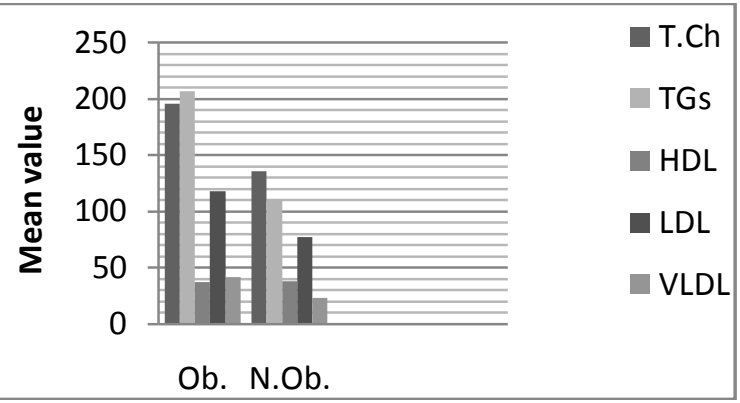

Fig 3. Mean fasting lipid profile in obese and non obese subjects.

\section{Discussion}

A relationship between periodontal disease and obesity has been suggested in recent reports. However, the first reported correlation between obesity and periodontal disease was found by Perlstein et al. in obese rats. (Perlstein et al. 1977). ${ }^{[13]}$ For the first time, in Japanese population Saito et al. found such an association in humans (Saito et al.1998,). ${ }^{[14]}$ Studies have also indicated that the fat distribution pattern plays a crucial role in the association with periodontitis (Saito et al., 2001; Al-Zahrani et al., 2003; Wood et al., 2003). ${ }^{[9,15-16]}$ In another study,Saito et al. concluded that obesity is associated with deep periodontal pockets, independent of glucose tolerance status (Saito et al., 2005). ${ }^{[17]}$ In USA using NHANES III data, Genco et al. demonstrated that BMI was positively correlated with the severity of periodontal attachment loss and found that this relationship is modulated by insulin resistance. ${ }^{[6]}$ Evidence for other populations, however is lacking.

Results of the present pilot study have shown significant difference in gingival index between both the groups. There is higher probing depth and loss of attachment in obese group compared to the non obese group but there was no statistically significant difference. These findings are in accordance with the study done by AlZahrani et al (2003). ${ }^{[15]}$ In fasting lipid profile the total cholesterol, triglycerides, LDL levels, VLDL levels were significantly higher in obese group. This is in accordance with the study done by Manjunath N. et al (2012). ${ }^{[18]}$ The mechanism by which obesity may affect the periodontium has not yet been determined. Studies in animals and humans suggest that over nutrition is associated with immune competence alterations. ${ }^{[2]}$

The adipose tissue is a complex and metabolically active endocrine organ that secretes numerous immunomodulatory factors(more than 50 bioactive molecules called adipokines)and plays a major role in regulating metabolic and vascular biology. ${ }^{[19]}$ Recent studies have also suggested that high serum levels of adipocytokines (Adiponectin, Resistin), leptin and IL-6, are associated with individuals presenting obesity and periodontitis. These findings indicate that obesity and periodontitis can, independently or jointly, alter the local and systemic levels of adipocytokines leading to the vicious cycle of events seen in these individuals. ${ }^{[20]}$ The plasminogen -activating system has been shown to play an important role in gingival inflammation. Plasminogen activator inhibitor -1(PAI-1) has an increased gene expression in visceral fat and induces agglutination of blood, may decrease periodontal blood flow in obesity, promoting initiation of periodontitis.TNF- $\alpha$ released by adipose tissue has been reported to cause liver injury in obese patients thus hepatic dyslipidemia. All these are the probable mechanisms underlying the periodontitis - dyslipidemia relationship in obese individuals. ${ }^{[16] .}$

\section{Conclusion}

The present pilot study has shown significant differences in gingival index, waist circumference, body mass index, total cholesterol, triglycerides and LDL levels, between obese and non obese group. The probing depth and loss of attachment are higher in obese group compared to the non obese group; however this difference was not found to be statistically significant. Further long term studies involving larger sample size are required to confirm the association between obesity and periodontal disease.

\section{Acknowledgements}

I express my gratitude to Dr. Rajesh H. (Dept. of Periodontics) and Dr. Sheriff M.H. (Dept. of Pathology) for the guidance and help. 


\section{References}

[1]. Ling Yang, Ping Li, Suneng Fu, Ediz S. Calay, Go"khan S. Hotamisligil. Defective Hepatic Autophagy in Obesity Promotes ER Stress and Causes Insulin resistance. Cell Metabolism. 2010; 11:467-478.

[2]. Dalla Vecchia CF, Susin C, Rösing CK, Oppermann RV, Albandar JM. Overweight and obesity as risk indicators for Periodontitis in Adults. J. Periodontol.2005; 76:1721- 1728.

[3]. Nield-Gehrig JS, Willmann DE. Foundations of periodontics for the dental hygienist (Lippincott Williams \& Wilkins, 2007).

[4]. Al - Zahrani MS, Bissada NF, Borawskit EA.Obesity and periodontal disease in young, middle-aged and older adults. J Periodontol. 2003; 74:610-615

[5]. Beck JD, Offenbacher S. Systemic effects of periodontitis: epidemiology of periodontal disease and cardiovascular disease. J Periodontol. 2005; 76(11):2089-2100.

[6]. Genco RJ, Grossi SG, Ho A, Nishimura F, Murayama Y. A proposed model linking inflammation to obesity, diabetes, and periodontal infections. J Periodontol. 2005; 76(11):2075-2084.

[7]. Beck J, Garcia R, Heiss G, Vokonas P, Offenbacher S. Periodontal disease and cardiovascular disease. J Periodontol. 1996; 67(10):1123-1137.

[8]. Loos BG. Systemic markers of inflammation in periodontitis. J Periodontol.2005; 76(11):2106-2115.

[9]. Saito T, Shimazaki Y, Koga T, Tsuzuki M, Oshima A. Relationship between upper body obesity and periodontitis. J Dent Res.2001; $80: 1631-1636$

[10]. Nishimura F, Iwamoto Y, Mineshiba J, Shimizu A, Soga Y, Murayama Y. Periodontal disease and diabetes mellitus: the role of tumor necrosis factor-alpha in a 2-way relationship. J Periodontol.2003; 74:97-102.

[11]. A Misra, P Chowbey, BM Makkar, NK Vikram, JS Wasir, D Chadha, et al. Consensus Statement for Diagnosis of Obesity, Abdominal Obesity and the Metabolic Syndrome for Asian Indians and Recommendations for Physical Activity, Medical and Surgical Management. J Assoc Physicians India. 2009; 57:163-170.

[12]. Loe H. The Gingival Index, the Plaque Index and the Retention Index Systems. J Periodontol.1967; 38(6):610-616.

[13]. Perlstein MI, Bissada NF. Influence of obesity and hypertension on the severity of periodontitis in rats. Oral Surg Oral Med Oral Pathol.1977; 43:707-719.

[14]. Saito T, Shimazaki Y, Sakamoto M. Obesity and periodontitis. N Eng J med.1998; 339:482-483.

[15]. Al - Zahrani MS, Bissada NF, Borawskit EA. Obesity and periodontal disease in young, middle-aged and older adults. J Periodontol. 2003; 74:610-615

[16]. Wood N, Johnson RB, Stereckfus CF. Comparison of body composition and periodontal disease using nutritional assessment technique: Third national health and nutritional examination survey (NHANES III). J Clin Periodontol. 2003; 30: $321-327$.

[17]. Saito T, Shimazaki Y, Kiyohara Y, Kato I, Kubo M, Iida M, et al. Relationship between obesity, glucose tolerance and periodontal disease in Japanese women: the hishayama study. J Periodontal Res. 2005; 40:346-353.

[18]. Manjunath N, Sahana.Comparison of plasma lipid and blood glucose level in obese and non obese patients with moderate periodontitis. Int Jour Contemp Dent. 2012; 3(1):63-67.

[19]. Ritchie C S. Obesity and periodontal disease. Periodontology 2000. 2007; 44:154-163.

[20]. Zimmermann GS, Bastos MF, Dias Gonçalves TE, Chambrone L, Duarte PM. Local and circulating levels of adipocytokines in obese and normal weight individuals with chronic periodontitis. J Periodontol. 2013; 84(5):624-633. 